\title{
Safety first: messages from Digestive Disease Week 2006
}

\author{
Stephen B Hanauer
}

Shortly after returning from Digestive Disease Week I was asked by a colleague what I thought were the most important messages from this year's meeting. In reply, I noted that before the meeting I had not gained the impression that many 'breakthrough' clinical science concepts were going to be presented, although incremental progress is evident on many research fronts. During the week before DDW, however, a confluence of events began to focus attention on the evolving research in the arena of risk management, particularly in relation to the role of biologic therapies for inflammatory bowel disease, and this theme continued to develop throughout the meeting.

The VIOXX ${ }^{\circledR}$ (rofecoxib; Merck \& Co.) story of the coronary complications of cyclo-oxygenase 2 inhibitors continues to unfold in the US courts. Meanwhile, final safety results from the celecoxib polyp-prevention trial were presented at DDW by N Arber et al. that described a small, potential cardiovascular risk associated with taking celecoxib for polyp chemoprevention. Yet more controversial are the reports of serious infections and neoplasia in rheumatoid arthritis patients treated with anti-tumor necrosis factor (TNF) agents (JAMA [2006] 295: 2275-2285), and the almost-simultaneous announcements that a rare form of hepatosplenic natural killer T-cell lymphomas can occur in young individuals treated with infliximab and/or azathioprine (Remicade ${ }^{\circledR}$ package insert), alongside the approval of infliximab therapy for children with Crohn's disease (Remicade ${ }^{\circledR}$; Centocor press release 19 May 2006). After nearly 8 years of marketing experience with infliximab, and while approval is pending for additional biologic therapies targeting TNF (and adhesion molecules), safety concerns have now clearly come to the forefront.

Biologic therapy has already been accepted for patients with rheumatoid arthritis, as a disease-modifying treatment that can prevent $\ldots$... DDW...

focused

attention

on risk

management

and patient

selection...

SB Hanauer is Editorin-Chief of Nature Clinical Practice Gastroenterology \& Hepatology.

Competing interests

The author declared he has no competing interests.

www.nature.com/clinicalpractice doi:10.1038/ncpgasthep0553 the early development of joint erosions. Yet, as we contemplate 'top-down' therapeutic approaches to the treatment of inflammatory bowel disease, appropriate patient selection for more-aggressive therapies becomes essential. At present, however, we have few selection criteria to determine who should receive early intervention with aggressive immunomodulation. Furthermore, while pediatricians have accepted the value of early therapy with thiopurine antimetabolites and concurrent corticosteroid intervention, how will we combine biologic therapy with immunosuppressive agents?

As with any therapeutic decision, the potential reward must outweigh the potential risk. For example, of how great a benefit is the modest reduction in polyps provided by celecoxib compared with a small risk of cardiovascular events over a 2-year period? How should we counsel young patients who have a chronic disease in relation to the substantial quality-of-life benefits provided by biologic or immunomodulatory therapies, versus the small possibility of developing infectious or neoplastic sequelae?

We have made great strides in the therapeutic effectiveness of biologic agents but there is still no such thing as a 'free lunch'. We have an increasing armamentarium available for the treatment of serious, chronic, life-altering but not life-threatening diseases. There is much to gain from using these therapies, but, while anchored to our "primum non nocere" covenant, the development of genetic and other biomarkers and other predictive and prognostic capabilities has lagged behind the expanding treatment options. My impression is that DDW not only highlighted basic and clinical scientific discoveries, but focused attention on risk management and patient selection to optimize the potential benefits of novel therapeutic approaches. 